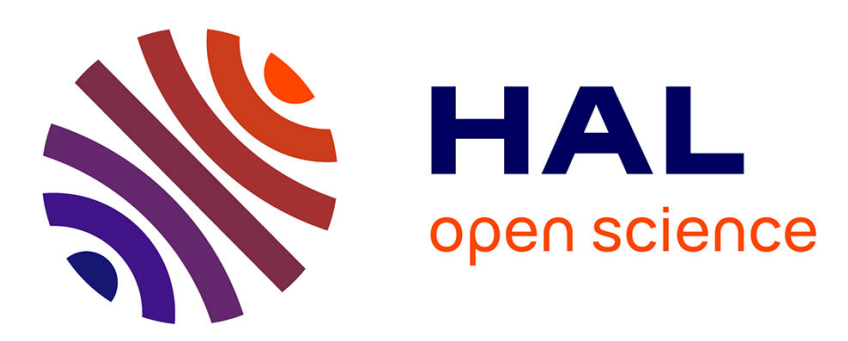

\title{
A Mass-flow Based MILP Formulation for the Inventory Routing with Explicit Energy Consumption
}

\author{
Yun He, Cyril Briand, Nicolas Jozefowiez
}

\section{To cite this version:}

Yun He, Cyril Briand, Nicolas Jozefowiez. A Mass-flow Based MILP Formulation for the Inventory Routing with Explicit Energy Consumption. International Conference on Operations Research and Enterprise Systems (ICORES 2016), Feb 2016, Rome, Italy. 10.5220/0005698802420251 . hal01282290

\section{HAL Id: hal-01282290 \\ https://hal.science/hal-01282290}

Submitted on 3 Mar 2016

HAL is a multi-disciplinary open access archive for the deposit and dissemination of scientific research documents, whether they are published or not. The documents may come from teaching and research institutions in France or abroad, or from public or private research centers.
L'archive ouverte pluridisciplinaire HAL, est destinée au dépôt et à la diffusion de documents scientifiques de niveau recherche, publiés ou non, émanant des établissements d'enseignement et de recherche français ou étrangers, des laboratoires publics ou privés. 


\title{
A Mass-flow Based MILP Formulation for the Inventory Routing with Explicit Energy Consumption
}

\author{
Yun $\mathrm{He}^{1,2}$, Cyril Briand ${ }^{1,2}$ and Nicolas Jozefowiez ${ }^{1,3}$ \\ ${ }^{1}$ CNRS, LAAS, 7 avenue du colonel Roche, F-31400 Toulouse, France \\ ${ }^{2}$ Univ de Toulouse, UPS, LAAS, F-31400 Toulouse, France \\ ${ }^{3}$ Univ de Toulouse, INSA, LAAS, F-31400 Toulouse, France \\ \{yunhe, briand, nicolas.jozefowiez\}@laas.fr
}

\begin{abstract}
Keywords: Inventory Routing Problem, Energy Minimization, Mixed Integer Linear Programming
Abstract: $\quad$ In this paper, we present a new mass-flow based Mixed Integer Linear Programming (MILP) formulation for the Inventory Routing Problem (IRP) with explicit energy consumption. The problem is based on a multiperiod single-vehicle IRP with one depot and several customers. Instead of minimizing the distance or inventory cost, the problem takes energy minimization as an objective. In this formulation, flow variables describing the transported mass serve as a link between the inventory control and the energy estimation. Based on physical laws of motion, a new energy estimation model is proposed using parameters like vehicle speed, average acceleration rate and number of stops. The solution process contains two phases with different objectives: one with inventory and transportation cost minimization as in traditional IRP, the other with energy minimization. Using benchmark instances for inventory routing with parameters for energy estimation, experiments have been conducted. Finally, the results of these two solution phases are compared to analyse the influence of energy consumption to the inventory routing systems.
\end{abstract}

\section{INTRODUCTION}

Our purpose is to introduce an energy estimation method and to propose a MILP optimization model that incorporates energy consumption into the IRP with mass flows. We start from a general literature review of the IRPs and the emerging Green Vehicle Routing Problems (GVRPs), then we discuss the incorporation of these two problems to present our new formulation.

The Vendor Managed Inventory (VMI) is an inventory management model where the supplier monitors the inventory level of the whole system and acts as a central decision maker for the long-term replenishment policy of each retailer. With respect to the traditional Retailer Managed Inventory (RMI), the VMI results in a more efficient resource utilization (?). Under the context of the VMI, the IRP combines the inventory management, vehicle routing and scheduling. There are three simultaneous decisions to make (?):

1. when to serve a customer;

2. how much to deliver when serving a customer;

3. how to route the vehicle among the customers to be served.

The IRP was first studied under the context of the distribution of industrial gases (?). After that, numbers of variants of the problem have come out, but there exists no standard version. The trade-offs between inventory costs and transportation costs are studied and explicit formulas to evaluate these tradeoffs based on spatial density of customers are obtained in (?). In (?), the authors proposed a method to reduce the long-term version of the problem to a single-period problem by defining single-period costs that reflects long-term effects. In (?), the authors tried to decide the long-run replenishment strategies for a set of geographically dispersed retailers and they proposed the first clustering algorithm for the IRP. In (?), the authors studied the inventory routing with continuous moves with both pick-ups and deliveries and developed a randomized greedy algorithm. The inventory routing with backlogging is studied in (?), where a heterogeneous fleet is considered. In (?), the authors proposed a MILP formulation of the IRP and applied a Branch-and-Cut algorithm to solve this problem. Later in (?), based on the formulation in the previous paper, new formulations are developed and 
compared with existing formulations using a large set of benchmark instances.

Two important literature reviews are worth mentioning here. (?) is a survey of the industrial aspects of the problem, and (?) is a comprehensive review on the typologies of the problem as well as their solution methods.

Nowadays, the Supply Chain Management is faced with a new challenge-the sustainability. As one of the three bottom lines of Sustainable Supply Chain Management, environmental sustainability is the most recognized dimension (?). As shown in (?), energy costs account for about $60 \%$ of the total cost of a unit of cargo transported on road.

In the literature, there are a growing number of papers about the green logistics and sustainable supply chain management. However, most of them treat the inventory management and the vehicle routing separately. The Energy Minimizing Vehicle Routing Problem (EMVRP) is studied in (?), where the Capacitated Vehicle Routing Problem (CVRP) is extended with a new cost function based on distance and load of the vehicle. In (?), a Fuel Consumption Rate (FCR) considered CVRP is proposed to minimize the fuel consumption. Focusing on the pollution and $\mathrm{CO}_{2}$ emission generated by the road transport sector, the Pollution Routing Problem (PRP) is proposed to explicitly control the Greenhouse Gas (GHG) emission of the transportation (?). The only paper that incorporates environmental aspects in the IRP is a case study from the petrochemical industry and total $\mathrm{CO}_{2}$ emissions are considered as a cost in the objective function (?).

A detailed literature review of the GVRP can be found in (?). In this review, the environmental sensitive Vehicle Routing Problem is divided into three groups: the Green-VRP for the optimization of energy consumption; the PRP for the reduction of pollution, especially GHG emissions; and the Vehicle Routing in Reverse Logistics for the wastes and end-of-life product collection. The authors point out that incorporating inventory models with PRP models can be promising.

This brief survey indicates that there lacks energy awareness in the inventory routing, or there lacks a system wide view in the sustainable routing. As the IRP integrates several decisions for the inventory control of the whole system, it helps us to make strategic decisions for energy optimization. First, under the VMI policy, the customer demands are flexible and can be distributed in different combinations. This property allows us to determine an optimal set of delivery quantities that yields the most environmental cost effectiveness while making sure that stock-out never happens. Second, the order of visit and the vehicle routes are to be determined. It is thus possible to design a routing strategy that takes the roads with the least energy costs. Third, the time of visit is also adaptable. We can choose a delivery time that is both convenient for the customers and that can also avoid rush hours, as congestion is one of the causes of high energy consumption and $\mathrm{CO}_{2}$ emissions.

Our study focuses on the integration of the IRP with explicit consideration on the energy consumption in the transportation. The main contributions of this paper are: (i) to propose an approach to estimate the energy consumed in the transportation activities of inventory routing; (ii) to reformulate the IRP to explicitly incorporate the energy consideration; (iii) to analyse the possible energy savings and the trade-offs between energy savings, travelled distances and inventory costs.

The remainder of this paper is organized as follows: in Section ??, the problem is described in details. Then, the mathematical model is presented in Section ??. After that, experimentation and results are given in Section ??, followed by the conclusion in Section ??.

\section{PROBLEM STATEMENT}

The problem in our study is based on a multiperiod single-vehicle deterministic inventory routing problem with one depot and several customers. The vehicle can leave the depot only once per period. In each period, it makes a tour around the customers that need to be refilled and returns to the depot. Stock-out and back-orders are not allowed in the model. Instead of the distance and inventory minimization, we take energy minimization as objective. Both the Maximum Level (ML) and the Order-up-to Level (OU) policy are applied to see the influence of different replenishment strategies to the energy consumption. A detailed explanation of these policies is given in Section ??

To facilitate the energy estimation, two units are used to measure inventory components - the number of components and the weight in kilograms $(\mathrm{kg})$. The number of components is used by the customers to represent their inventory levels and to count the number of packages of delivered goods. The weight is used by the transporters. It is the physical mass of the components transported by the vehicle.

The next parts present in details the parameters and variables of the problem and the computation of the energy estimation function. In particular, mass flow variables are introduced to link energy estima- 
tion and inventory management.

\subsection{General Settings for Routing}

The problem is constructed on a complete undirected graph $\mathcal{G}=\{V, E\} . V=\{0, \ldots, n\}$ is the vertex set. It includes one depot denoted by 0 and the customers to visit denoted by the set $V_{c}=\{1, \ldots, n\} . E=\{(i, j) \mid$ $i, j \in V$ and $i<j\}$ is the set of undirected edges. There are $T$ replenishment planning periods. Each period can be a day, a week or even a month according to the real situation. In each period, only one tour can be performed. If a tour is presented in a period, the vehicle starts from the depot, travels through all the customers who need to be served at this period and returns back to the depot at the end of the period.

Three kinds of decision variables $z_{i}^{t}, x_{i j}^{t}$ and $y_{i j}^{t}$ correspond to routing. For each $i \in V_{c}, t \in T, z_{i}^{t}$ is a binary variable indicating whether customer $i$ is served at period $t$. It equals 1 if customer $i$ is served and 0 otherwise. Particularly, $z_{0}^{t}$ indicates whether the tour at period $t$ is performed (equals 1 ) or not (0). For each edge $(i, j) \in E$ and each period $t \in T, x_{i j}^{t}$ is an integer variable indicating the number of times that edge $(i, j)$ is used in the tour of period $t$. For each arc $(i, j) \in V \times V$ and each period $t \in T$, variable $y_{i j}^{t}$ is a binary variable to indicate the direction of the vehicle route. It equals 1 if the vehicle travels from node $i$ to $j$ at period $t$ and 0 otherwise.

The vehicle has a capacity $Q$ expressed in numbers of components and a mass limit $M$. The empty vehicle mass, or curb weight $(\mathrm{kg})$ of the vehicle is $W$.

\subsection{Inventory Characteristics}

Inventory levels at customers and depot are monitored during the whole planning time horizon. They are summarised at the end of each replenishment period. The customer demands are described as demand rates per period. In each period, $r_{i}$ units of components are demanded by customer $i \in V_{c}$. In particular, $r_{0}$ is the number of components made available at the depot in each period. Each customer $i \in V_{c}$ has a stocking capacity $C_{i}$ while the depot is supposed to have an unlimited stocking capacity. $h_{i}$ is the inventory storage cost per unit of component per period at customer $i$ or the depot. The weight of a component in kilograms at customer $i \in V_{c}$ is denoted by $m_{i}$.

Two variables are defined for the inventory management. The variable $I_{i}^{t}$ is the inventory level in number of components at the depot 0 or at the customer $i \in V_{c}$ at the end of period $t$. The variable $q_{i}^{t}$ is the number of components delivered to customer $i \in V_{c}$ during period $t \in T$.

\subsection{Energy Estimation}

There are many complicated models in the literature that estimate the $\mathrm{CO}_{2}$ emissions or the amount of pollutants produced by a vehicle on road. (?) is a review of vehicle emission models and their inclusion into the existing optimization methods. However, few models focus on the energy consumed and most of them depend on vehicle type. This can be partly explained by the fact that the main power source of vehicles used today is petroleum. Nevertheless, with the emergence of electric and hybrid vehicles, we find it more appropriate to estimate the energy consumption instead of fuel consumption. In addition, for the generality of the problem, it is important that the model would apply for every type of vehicles. According to (?), travelling kinematic variation (accelerations, idle duration, etc.) obviously affects engine load and by turns the energy consumption. Thus, in this paper, we propose a general simple model based on vehicle dynamics. This model would be applicable to European suburban transportation network with short or medium distances and potentially high traffic intensity. It can give us a gross estimation of the energy required by a vehicle on a road segment with speed variation, independent of vehicle type or energy source.

In our model, the stop rate $\tau$, i.e., the number of stops per unit of distance is used to model the dynamics of the vehicle on a fixed segment of road. This parameter can also represent the traffic condition on the road. More precisely, with a traffic near free flow, $\tau$ takes a value near 0 , which means that the vehicle goes through the road fluently without any stops; however, with congestion, this number is set to a bigger value to indicate a frequent speed variation. Usually $\tau$ takes a value between 0 and 4 depending on road types (?). Moreover, there exists an interrelationship between the distance travelled, the stop rate and the speed and acceleration of the vehicle, which is generally explained in Section ?? and is not the subject of our study.

Suppose a vehicle travelling from one location to another. The path of the vehicle between two locations is supposed to be predefined with an average stop rate $\tau$, and the total distance travelled is $s$. So the vehicle stops $\tau \cdot s$ times during the trip. The coefficient of friction is a fixed parameter $\mu=0.01$. The gravitational acceleration is $g=9.81 \mathrm{~m} / \mathrm{s}^{2}$. The environmental effects of the road (wind, temperature etc.) as well as the viscosity of air are ignored. Road slopes are also ignored for the moment but will be considered in the future. As a result, the only forces exerted on the vehicle are the rolling resistance and the traction.

Between every two stops, the vehicle speed is 


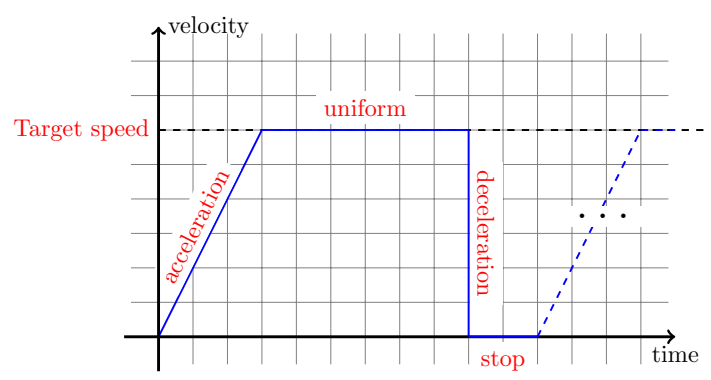

Figure 1: The speed variation of the vehicle with time

supposed to follow a fixed pattern of variationacceleration, uniform speed movement and stop (Figure ??). Each time, the vehicle speeds up from 0 to the target speed $V$ with a fixed acceleration $a_{\text {acc }}$. It goes on at this speed for a while and then stops. The stop is supposed to be instantaneous. This pattern is repeated $\tau \cdot s$ times supposing that the vehicle has no speed at both the starting and the ending point. After each stop, it speeds up again to the same target speed.

The next part explains how to estimate energy consumption using this simple model.

The mathematical relationship between the physical quantities of energy $(E)$, work $(W)$ and power $(P)$ are:

$$
\begin{aligned}
E & =\int P(t) d t \\
P(t) & =F v(t) \\
E & =W=\sum F s
\end{aligned}
$$

with $F$ a constant force, $v(t)$ the speed at instant $t$ and $s$ the distance travelled.

According to knowledge of physics and energy conservation, under the hypothesis of speed variation presented above, the total energy cost per unit of mass when distance $s$ is travelled with stop rate $\tau$ is then:

$$
c=g \mu s+\tau s V^{2}
$$

Figure ?? shows the power variation of the vehicle under the previous speed variation. We can see that each time the vehicle speeds up, there appears a "peak" of engine power which corresponds to a potentially high energy consumption. This is also reflected by Equation ?? - the more the vehicle stops on a road segment ( $\tau$ takes a bigger value), the higher the energy would cost. See the Appendix for the detailed calculation.

In this way, we define $c_{i j}=g \mu s_{i j}+\tau_{i j} s_{i j} V_{i j}^{2}$ the energy cost per unit of mass for each edge $(i, j)$. It is related to the distance travelled $s_{i j}$ and the dynamics of the vehicle on the road as expressed by the stop rate $\tau_{i j}$ and target speed $V_{i j}$

If $m_{i j}(\mathrm{~kg})$ of mass is loaded on the vehicle when traversing from $i$ to $j$ and the vehicle weighs $W(\mathrm{~kg})$,

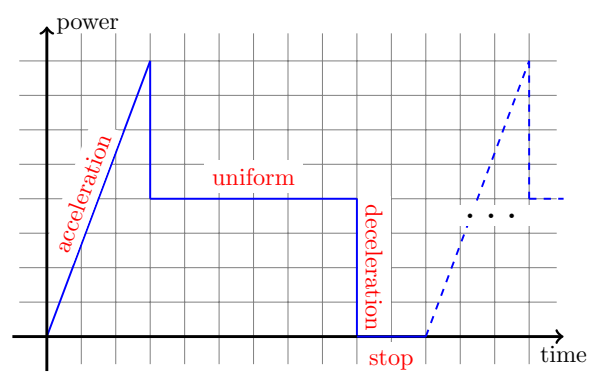

Figure 2: The power variation with time

the energy cost of the vehicle travelling from $i$ to $j$ is thus:

$$
c_{i j}\left(m_{i j}+W\right)
$$

\subsection{Commodity Mass Flow}

As we can see from the energy estimation computation, the total energy cost is a linear function of mass. Meanwhile, the mass or the quantity of products is also an important element in the inventory management. It is a measurement of the inventory levels. In fact, there exists a mass flow inside the transportation network and it can serve as a bridge linking the inventory routing and the energy optimization.

In the traditional IRP formulations presented in (?), a flow formulation exists to model the inventory flows inside the transportation network. Our model takes advantage of this formulation. Instead of considering the flow in terms of number of components, the mass of the shipped components is considered. Once we decide the mass transported on each edge of the network at each period, we can deduce the number of components leaved at each customer vertex. Or inversely, if we know how many units of components are delivered to each customer at each period, we can decide the order of visits and get a flow of mass in the transportation network that minimizes the energy consumed.

In our model, variables $m_{i j}^{t}$ are defined as the mass transported by the vehicle from $i$ to $j$ at period $t$. They are linked with the vehicle flow variables $y_{i j}^{t}$. If the vehicle does not go from $i$ to $j$ at period $t\left(y_{i j}^{t}=0\right)$, $m_{i j}^{t}$ is equal to 0 .

Figure ?? details the various flows traversing customer $i$ at period $t$. The inventory flow $I_{i}^{t}$ and the demand $r_{i}$, expressed in number of components, are associated with the dotted arcs. They describe the variation of the inventory level of $i$ with time periods. The solid arcs stand for the mass of the incoming and outgoing products ( $m_{j i}^{t}$ and $m_{i j}^{t}$ respectively). They are used to estimate the potential energy consumption, with $c_{i j}^{t}$ the energy cost per unit of mass on edge $(i, j)$. 


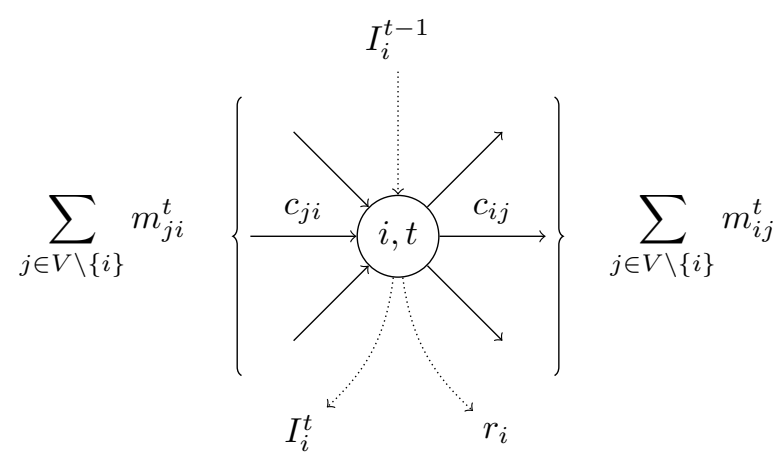

Figure 3: The flows passing through customer $i$ at period $t$

The difference $\frac{1}{m_{i}}\left(\sum_{j \in V \backslash\{i\}} m_{j i}^{t}-\sum_{j \in V \backslash\{i\}} m_{i j}^{t}\right)$ gives the number of components $q_{i}^{t}$ delivered by the vehicle to customer $i$ during period $\mathrm{t}$.

\section{MATHEMATICAL MODEL}

In this section, we present the mathematical model using the mass flow.

\subsection{Objectives}

Two objectives are defined, one for inventory and distance optimization and the other for energy optimization. The objective function (??) is the traditional one as in (?). It is the sum of the total distance travelled plus the sum of the inventory storage costs over all the periods.

$$
\min \sum_{t \in T} \sum_{(i, j) \in V \times V} s_{i j} y_{i j}^{t}+\sum_{t \in T} \sum_{i \in V} h_{i} I_{i}^{t}
$$

The objective function (??) is the sum of the total energy consumed in the inventory routing over all the periods. Note that it contains two terms: one is a flexible cost related to the transported mass of the vehicle $m_{i j}^{t}$, and the other is a fixed cost induced by the vehicle curb weight $W$.

$$
\min \sum_{t \in T} \sum_{(i, j) \in V \times V} c_{i j} m_{i j}^{t}+W \sum_{t \in T} \sum_{(i, j) \in V \times V} c_{i j} y_{i j}^{t}
$$

\subsection{Constraints}

Here are the sets of constraints. Compared with the basic flow formulation in (?), mass flow variables take place of commodity flow variables.

\section{Inventory Management}

Constraints (??) to (??) are for monitoring the inventory levels of each location at each period.

$$
\begin{array}{lrl}
I_{0}^{t}=I_{0}^{t-1}+r_{0}-\sum_{i \in V_{c}} q_{i}^{t} & \forall t \in T \\
I_{i}^{t}=I_{i}^{t-1}-r_{i}+q_{i}^{t} & \forall i \in V_{c}, t \in T \\
q_{i}^{t} \geq C_{i} z_{i}^{t}-I_{i}^{t-1} & \forall i \in V_{c}, t \in T \\
q_{i}^{t} \leq C_{i}-I_{i}^{t-1} & \forall i \in V_{c}, t \in T \\
q_{i}^{t} \leq C_{i} z_{i}^{t} & & \forall i \in V_{c}, t \in T
\end{array}
$$

Constraints (??) and (??) ensure that the inventory levels of each station are coherent from one period to another. The OU inventory policy is ensured by constraints (??) and (??)—after each delivery, the inventory level of each visited customer is fulfilled to the maximum. If we delete Constraints (??), the model becomes one under the ML policy, where the replenishment level is flexible but bounded by the stocking capacity of each customer. Constraints (??) ensure that if a customer $i$ is not visited at a period $t\left(z_{i}^{t}=0\right)$, the delivered quantity $q_{i}^{t}$ equals 0 and if the customer is visited, the delivered quantity never exceeds the capacity.

\section{Commodity Mass Flow Management}

Constraints (??) and (??) are the mass flow constraints.

$$
\begin{array}{rlrl}
\sum_{j \in V_{c}} m_{0 j}^{t} & =\sum_{i \in V_{c}} q_{i}^{t} m_{i} & \forall t & \in T \\
\sum_{j \in V} m_{j i}^{t}-\sum_{j \in V} m_{i j}^{t} & =q_{i}^{t} m_{i}
\end{array} \quad \forall i \in V_{c}, t \in T
$$

Constraints (??) ensure that at period $t$, the mass out of the depot is equal to the total mass transported to all the customers. Constraints (??) ensure that for each customer $i$ at each period $t$, the quantity received is equal to the difference between the entering and the leaving mass flow.

\section{Vehicle Routing}

Constraints (??) to Constraints (??) are typical routing constraints.

Undirected routing

$$
\begin{aligned}
& \sum_{j \in V_{c}} x_{0 j}^{t}=2 z_{0}^{t} \quad \forall t \in T \\
& \sum_{\substack{j \in V \\
j<i}} x_{j i}^{t}+\sum_{\substack{j \in V_{c} \\
j>i}} x_{i j}^{t}=2 z_{i}^{t} \quad \forall i \in V_{c}, t \in T
\end{aligned}
$$

\section{Directed vehicle flow}

$$
\sum_{j \in V_{c}} y_{0 j}^{t}=z_{0}^{t} \quad \forall t \in T
$$




$$
\begin{aligned}
\sum_{j \in V} y_{i j}^{t}=z_{i}^{t} & \forall t \in T, i \in V_{c} \\
\sum_{j \in V} y_{j i}^{t}=z_{i}^{t} & \forall t \in T, i \in V_{c} \\
x_{i j}^{t}=y_{i j}^{t}+y_{j i}^{t} & \forall t \in T,(i, j) \in E
\end{aligned}
$$

Constraints (??) and (??) define the non-directed route of the vehicle in each period. Constraints (??)(??) restrain the direction of the vehicle flow. They link $y$ and $z$ variables to make sure that in each period at most one tour is performed and that each customer is visited at most once in each period. Constraints (??) link variables $y$ and $x$ to ensure that each edge is used at most once in each period.

\section{Vehicle Capacity}

Constraints (??) and (??) guarantee that the vehicle capacity is never exceeded both in number of components and in unit of mass.

$$
\begin{array}{rr}
\sum_{i \in V_{c}} q_{i}^{t} \leq Q z_{0}^{t} & \forall t \in T \\
m_{i j}^{t} & \leq M y_{i j}^{t} \quad \forall t \in T,(i, j) \in V \times V
\end{array}
$$

Constraints (??) also link the mass flow and the vehicle flow on the graph. They make sure that the direction of the vehicle flow is the same as that of the mass flow.

\section{Variable Domains}

Constraints (??)-(??) are the variable domains.

$$
\begin{aligned}
0 \leq I_{i}^{t} & \leq C_{i}, I_{i}^{t} \in \mathbb{N} & \forall i \in V, t & \in T \\
0 \leq q_{i}^{t} & \leq Q, q_{i}^{t} \in \mathbb{N} & \forall i \in V_{c}, t & \in T \\
0 \leq m_{i j}^{t} & \leq M, m_{i j}^{t} \in \mathbb{N} & \forall(i, j) \in V \times V, t & \in T \\
z_{i}^{t} & \in\{0,1\} & \forall i \in V, t & \in T \\
x_{i j}^{t} & \in\{0,1\} & \forall(i, j) \in E, i<j, t & \in T \\
x_{0 j}^{t} & \in\{0,1,2\} & \forall j \in V_{c}, t & \in T \\
y_{i j}^{t} & \in\{0,1\} & \forall(i, j) \in V \times V, t & \in T
\end{aligned}
$$

All the variables take integer values. Note that for variables $x_{0 j}^{t}$, since direct routing is possible, they can be assigned with value 2 .

\subsection{Solution Methodology}

The solution process is divided into two phases. In the first phase, the objective is to minimize the combined cost of transportation and inventory as in objective function (??). In the second phase, starting with the solution of the first phase, the same model is solved to minimize the total energy consumption as computed in objective function (??).
The methodology allows us to quickly find a feasible solution for energy minimization in the first phase, and then explore the energy minimization possibilities in the second phase.

\section{EXPERIMENTATION}

This section explains the data generation method, gives the system settings and provides an analysis of the obtained results.

\subsection{Data Generation}

Based on existing IRP instances proposed in (?), new instances more adapted to energy estimation are generated. Information on stop rates $\tau$ and vehicle target speeds $V$ relative to the distance is added in the existing instances. The correlation within these parameters is determined based on empirical data of delivery trucks on real routes provided by (?). The following part explains how the data set is generated.

First, three types of road is considered-highway, national route and urban road. For each edge between two locations, the type of road is generated randomly. Then, target speed and number of stops for different types of roads are generated using different methods. Details of these methods are given in the Appendix. Finally, for all types of road, the average acceleration is fixed at $1.01 \mathrm{~m} / \mathrm{s}^{2}$.

Then, a random number between 1 and 10 is generated for each customer $i$ to represent the mass of a package of components $m_{i}$. Vehicle weight and mass capacity are correlated according to vehicle information provided in (?).

In total, 256 cases are generated. Each case contains 5 instances. The cases are categorized by the number of periods ( 3 or 6 periods of replenishment planning), the proportion of the inventory storage cost in relation to the transportation cost (high or low), the inventory replenishment policy (OU or ML), the proportion of each type of road in the whole map and the number of customers in the map.

\subsection{System Settings}

The model is realized in $\mathrm{C}++$ with $I B M^{\circledR} I L O G^{\circledR}$ CPLEX 12.6.1.0 and solved by the default Branchand-Bound algorithm with one thread. The operation system is Ubuntu 14.04 LTS with Intel ${ }^{\circledR}$ Core $^{\circledR}$ i7$47903.60 \mathrm{GHz}$ processor and $16 \mathrm{G}$ memory.

A time limit of 1800 seconds is set for each of the two phases. All the other settings of CPLEX are 
as default. The results of both of the two phases are compared in the next part.

\subsection{Result Analysis}

\section{Performance}

Eventually, within the time limit, nearly $70 \%$ of the instances with distance and inventory optimization can be solved to optimality by CPLEX, while only one third of the instances can be solved to optimality with energy minimization. Table ?? gives a summary of the performance information.

The dimension of the instance is determined by the number of periods ("T" column in the table) and the number of customers ("n" column in the table). The inventory policy (OU or ML) changes the constraint sets of the model. A combination of these three parameters define a category of instances. Each category contains 40 instances. In this table, computation time of each solution phase ("time1" and "time2") and the solution status within the time limit ("status1" and "status2") are presented. The times are average values over all the instances of the same category. If all the instances of this category can be solved to optimality by CPLEX, the status is noted "Optimal". Otherwise, the average relative gap after 1800 seconds of computation is reported.

As we can see from this table, energy minimization is much more difficult to solve than inventory and transportation cost minimization (time $2 \gg$ time1). This may result from the large possible combination of the values of the mass flows. It is normal to see that the problem becomes more difficult as the dimension of the instances increases. For both OU and ML policies, instances larger than 20 customers with 3 periods or 15 customers with 6 periods become impossible to solve for energy optimization (Phase 2) within the time limit. And it seems that the influence of the inventory policy to energy minimization is not as much as in the traditional IRP. Figure ?? and ?? show the solving time increase with the number of customers for different number of periods and inventory policies.

\section{Energy Reduction}

In general, $95 \%$ of all the instances can expect an energy reduction. Of all the instances where energy is reduced, the average reduction is $3.7 \%$, and the maximum reduction can reach $19.4 \%$. Meanwhile, the distance and inventory cost remains nearly the same (with an average augmentation rate of less than $1 \%$ ). In addition, the experiments show that nearly all of the instances with 6 periods can be assigned an energysaving replenishment strategy and nearly $90 \%$ of the

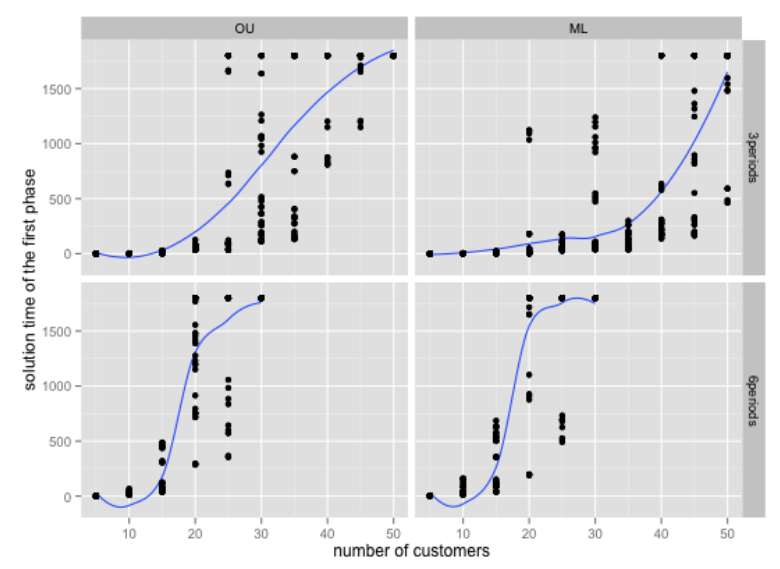

Figure 4: Solution time of the first phase

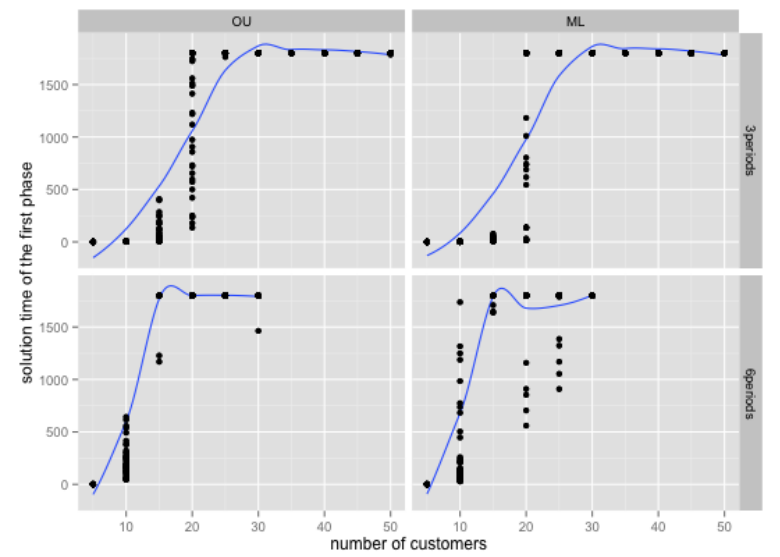

Figure 5: Solution time of the second phase

instances with 3 periods can achieve a solution with less energy consumption.

By inspecting the results in details, it is observed that most of the energy reduction comes from the transported mass, which correspond to the flexible part of the energy minimizing objective function. The energy reduction induced by the transported mass is nearly $20 \%$ in average and it can reach as high as $66.3 \%$. And by analysing the energy objective function, it is easy to see that minimizing the fixed part of the energy cost or the part induced by the curb weight is equivalent to a weighted minimization of the distance. Since the vehicle mass accounts for nearly half of the total mass moving on the road, a great part of the energy minimization is equivalent to distance minimization. This can explain the reason why finally the total energy reduction is so small even though the energy consumed by transporting the products can be highly reduced.

It should also be noted that the inventory policy 
Table 1: Performance summary of all the instances

\begin{tabular}{|l|l|llll|l|llll|}
\hline & \multicolumn{6}{|c|}{ OU policy } & \multicolumn{5}{c|}{ ML Policy } \\
\hline $\mathrm{T}$ & $\mathrm{n}$ & time1 & time2 & status1 & status2 & $\mathrm{n}$ & time1 & time2 & status1 & status2 \\
\hline & 5 & 0.0665 & 0.108 & Optimal & Optimal & 5 & 0.142 & 0.0759 & Optimal & Optimal \\
& 10 & 1.84 & 3.44 & Optimal & Optimal & 10 & 2.37 & 2.47 & Optimal & Optimal \\
& 15 & 14.9 & 83.8 & Optimal & Optimal & 15 & 10.0 & 23.6 & Optimal & Optimal \\
& 20 & 53.6 & 1235.4 & Optimal & 0.0228 & 20 & 138.8 & 1075.9 & Optimal & 0.0645 \\
3 & 25 & 642.7 & 1799.1 & 0.0114 & 0.0594 & 25 & 71.33 & 1800.0 & Optimal & 0.1053 \\
& 30 & 721.9 & 1800.0 & 0.0106 & 0.1028 & 30 & 307.1 & 1800.0 & Optimal & 0.1175 \\
& 35 & 1079.4 & 1800.0 & 0.0196 & 0.132 & 35 & 112.6 & 1800.0 & Optimal & 0.1362 \\
& 40 & 1624.6 & 1800.0 & 0.0336 & 0.1679 & 40 & 570.4 & 1800.0 & 0.0380 & 0.1564 \\
& 45 & 1729.6 & 1800.0 & 0.0572 & 0.2185 & 45 & 1043.3 & 1800.0 & 0.0233 & 0.1873 \\
& 50 & 1800.0 & 1799.6 & 0.0767 & 0.2260 & 50 & 1645.8 & 1800.0 & 0.0175 & 0.2108 \\
\hline & 5 & 0.694 & 1.194 & Optimal & Optimal & 5 & 4.021 & 1.243 & Optimal & Optimal \\
& 10 & 26.94 & 234.7 & Optimal & Optimal & 10 & 45.39 & 324.6 & Optimal & 0.0201 \\
6 & 15 & 177.2 & 1769.9 & Optimal & 0.0342 & 15 & 262.1 & 1789.8 & Optimal & 0.0465 \\
& 20 & 1307.7 & 1800.0 & 0.0372 & 0.1133 & 20 & 1545.3 & 1679.6 & 0.0180 & 0.1115 \\
& 25 & 1449.7 & 1800.0 & 0.0298 & 0.1349 & 25 & 1563.9 & 1720.7 & 0.0304 & 0.1501 \\
& 30 & 1800.0 & 1791.6 & 0.0453 & 0.1792 & 30 & 1800.0 & 1800.0 & 0.0416 & 0.1748 \\
\hline
\end{tabular}

has an influence on the energy minimization potential. Figure ?? and ?? show the relationship between distance (inventory) and energy reduction. The solid curves represent average values of all the instances. We can see that under the OU policy, most of the reduction is obtained by minimizing the distance travelled. The more energy reduction there is, the less distance would be travelled. Under the ML policy, most of the reduction is obtained by changing the inventory replenishment strategy. This could result from the fact that the ML policy is more flexible than the OU policy.

Figure ?? shows the energy reduction in relation to the number of customers under different policies. We can see that for small instances, ML policy tends to produce a lower cost, but with the increase of the dimension of instances, the model becomes more difficult to solve with ML policy than with OU policy, so for the most difficult instances where non-optimal solution is reached within the time limit, it is still OU policy that gives a better result. These results imply that inventory replenishment strategy is important for energy minimization. Instead of always fill the inventory to the maximum level as suggested by OU policy, ML policy may give a better energy solution.

We could not observe much relationship between road types and energy minimization. In fact, the influence of road types to the energy reduction becomes noticeable only for instances with large number of customers. This could result from a lack of realistic data. So more tests should be done with more realistic datasets. And the solution method should be improved so that realistic-sized instances could be solved to optimality to show the energy saving potential of different road types.

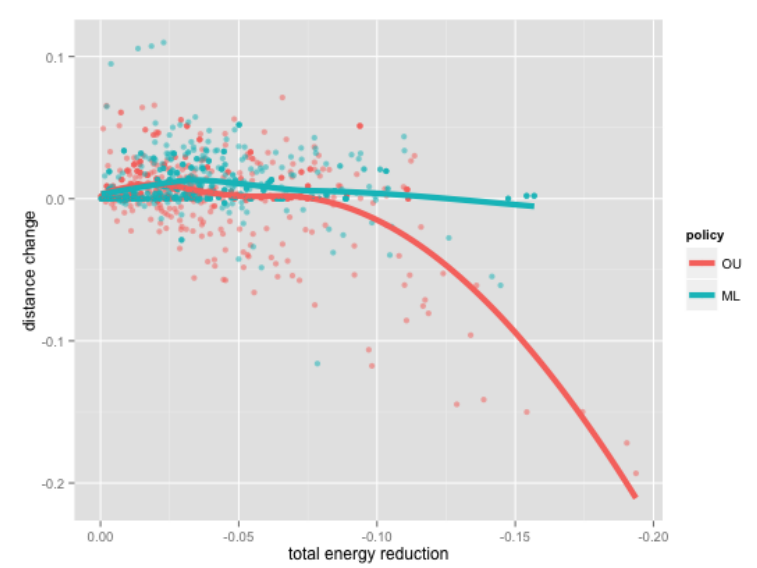

Figure 6: The relationship between distance and energy reduction

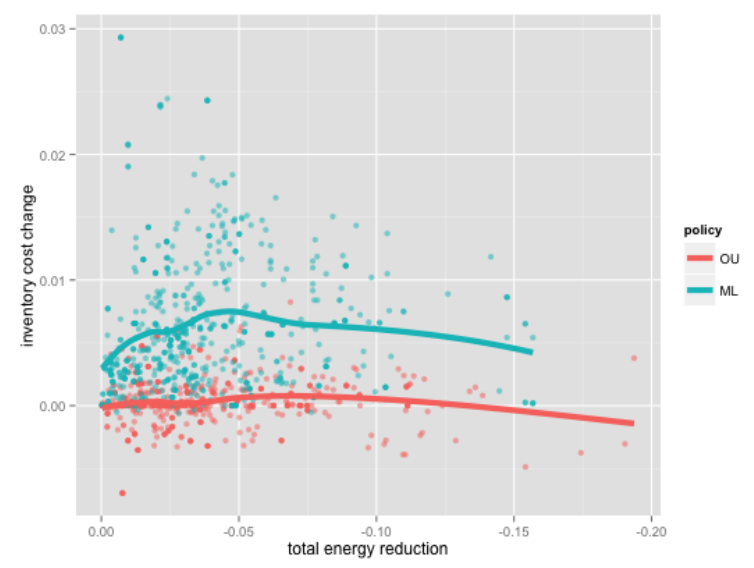

Figure 7: The relationship between inventory and energy reduction 


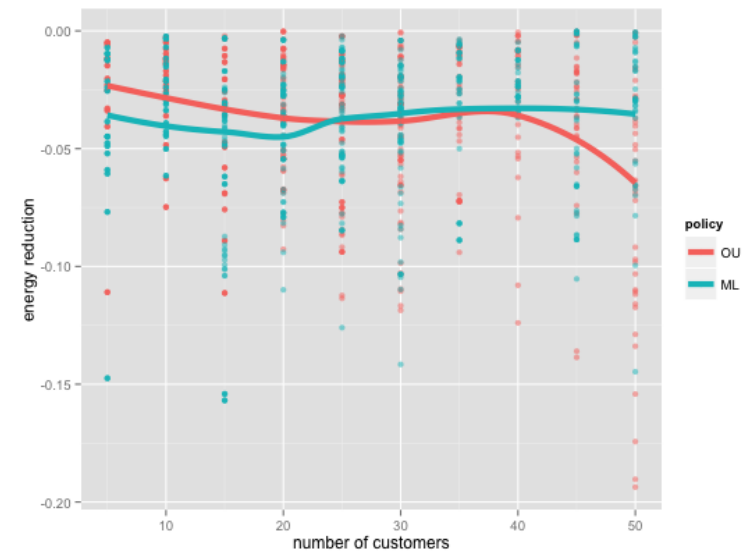

Figure 8: The relationship between energy reduction and number of customers under different inventory policy

\section{CONCLUSIONS}

Energy consumption is an important aspect in both economical and ecological view. It becomes more and more important with the sustainable requirement of the inventory systems. However, few researchers paid attention to the combination of inventory management, vehicle routing and energy minimization. This new mass flow-based formulation of the IRP with energy consumption addresses the problem explicitly. An energy estimation method is proposed that combines vehicle dynamics and road characteristics. This estimation gives us an energy cost function that is linear to the total mass. In this new IRP formulation, the mass is added as a decision variable and the energy cost function is considered as an objective. The relationship between the vehicle dynamics in the transportation network, the inventory management strategy and the energy consumption estimation is examined. Our first experimentation shows that a better energy cost can be achieved by adjusting the inventory replenishment planning. Among all the influence factors, the inventory policy is an important one.

Further works need to be done on the modelling of traffic networks, so that different road types, especially road slops, traffic conditions as well as vehicle speed levels could be considered in the decision process. The estimation of the energy costs needs to be more representative. More data are needed from the real world to accomplish the work. The inventory routing model needs to be improved to better control the time and quantity of each delivery. Solution algorithms and heuristics are to be explored to speed up the computation, especially with realistic data that would contain larger number of customers or longer decision periods. The extension of the problem to a multi-objective one is also a promising track of study. 


\section{APPENDIX}

\section{Energy Estimation}

According to knowledge of physics and energy conservation, under the hypothesis of speed variation presented in Section ??, we have:

- In each acceleration phase, the speed of the vehicle increases from 0 to the target speed $V$ with constant acceleration $a_{\text {acc }}$. With $F_{\text {acc }}$ the traction force of the engine, $s_{\text {acc }}$ the distance travelled, $E_{\text {acc }}$ the energy consumed, and $P_{\text {acc }}(t)$ the engine power:

$$
\begin{aligned}
v(t) & =a_{\mathrm{acc}} t \\
s_{\mathrm{acc}}(t) & =\frac{1}{2} a_{\mathrm{acc}} t^{2} \\
F_{\mathrm{acc}}-m g \mu & =m a_{\mathrm{acc}} \\
P_{\mathrm{acc}}(t)=F_{\mathrm{acc}} v(t) & \left.=m\left(a_{\mathrm{acc}}^{2}+g \mu\right) a_{\mathrm{acc}}\right) t \\
E_{\mathrm{acc}}-m g \mu s_{\mathrm{acc}} & =\frac{1}{2} m V^{2}
\end{aligned}
$$

At the end of this phase, the engine power is

$$
P_{\mathrm{acc}}=m\left(a_{\mathrm{acc}}+g \mu\right) V
$$

the distance travelled is

$$
s_{\mathrm{acc}}=\frac{V^{2}}{2 a_{\mathrm{acc}}}
$$

The total energy cost per unit of mass is

$$
c_{\mathrm{acc}}=g \mu s_{\mathrm{acc}}+\frac{1}{2} V^{2}
$$

- In the uniform-speed phase, the distance $s_{u}$ is computed as the difference between the total distance $s$ and the total distance travelled in acceleration and deceleration. Since the deceleration is considered to be instantaneous $\left(s_{\mathrm{dec}}=0\right)$, the total distance travelled at uniform speed is calculated as:

$$
s_{u}=s-\tau s\left(s_{\mathrm{acc}}+s_{\mathrm{dec}}\right)=s-\tau s s_{\mathrm{acc}}
$$

with $\tau \cdot s$ the total number of stops. The engine power is also constant

$$
P_{\mathrm{u}}(t)=F_{\mathrm{u}} V=m g \mu V
$$

The total energy cost per unit of mass in the uniform phase is:

$$
c_{u}=g \mu\left(s-\tau s s_{\mathrm{acc}}\right) .
$$

- In each deceleration phase, since we consider an instantaneous stop,

$$
s_{\mathrm{dec}}=0
$$

$$
\begin{aligned}
P_{\mathrm{dec}}(t) & =0 \\
E_{\mathrm{dec}} & =\frac{1}{2} m V^{2}
\end{aligned}
$$

and the energy cost per unit of mass is

$$
c_{\mathrm{dec}}=\frac{1}{2} V^{2}
$$

the total energy cost per unit of mass when distance $s$ is travelled with stop rate $\tau$ is then:

$$
c=c_{u}+\tau \cdot s\left(c_{\mathrm{acc}}+c_{\mathrm{dec}}\right) .
$$

Finally, we get:

$$
c=g \mu s+\tau s V^{2}
$$

\section{Calculation of Parameters for Different Road Types}

On a highway, the maximum speed is fixed at $110 \mathrm{~km} / \mathrm{h}$, and the number of stops is fixed at 2 stops per edge independent of the road distance travelled. On a national route, the vehicle speed is fixed at $80 \mathrm{~km} / \mathrm{h}$ and the number of stops is linearly dependent on the distance with a random error. On an urban road, both the stop rate and the vehicle speed are dependent on the distance travelled. The stop rate $\tau$ is determined by a linear function of the distance as in the case of national route. And the target speed $V$ is derived from the stop rate $\tau$ by an equation of the form $V=\beta+\alpha_{1} \cdot \tau+\alpha_{2} / \tau$. 